\title{
NOTE ON OSCILLATION THEOREMS FOR THIRD ORDER NON-LINEAR DIFFERENCE EQUATIONS
}

\author{
I. MOHAMMED ALI JAFFER AND B. SELVARAJ
}

\begin{abstract}
In this paper some sufficient conditions for oscillation of all solutions of certain difference equations are obtained. Examples are given to illustrate the results.
\end{abstract}

\section{Introduction}

We are concerned with the oscillatory properies of all solutions of third order linear and non-linear difference equations of the form

$$
\begin{array}{rlrl}
\Delta^{2}\left(a_{n} \Delta x_{n}\right)-p_{n} \Delta x_{n}+q_{n} f\left(x_{n+1}\right)=0, & & n=0,1,2 \ldots, \\
\Delta^{2}\left(a_{n} \phi\left(x_{n}\right) \Delta x_{n}\right)-p_{n} \Delta x_{n}+q_{n} f\left(x_{n+1}\right) & =0, & & n=0,1,2 \ldots, \\
\Delta^{2}\left(a_{n} \Delta x_{n}\right)+q_{n} f\left(x_{n+1}\right) & =0, & & n=0,1,2 \ldots, \\
\Delta^{2}\left(a_{n} \phi\left(x_{n}\right) \Delta x_{n}\right)+q_{n} f\left(x_{n+1}\right) & =0, & & n=0,1,2 \ldots,
\end{array}
$$

where the following conditions are assumed to hold.

(H1) $\left\{a_{n}\right\},\left\{p_{n}\right\}$ and $\left\{q_{n}\right\}$ are real positive sequences where $n \in N=\{0,1,2,3 ., \ldots\}$.

(H2) $f: R \rightarrow R$ is continous and $x f(x)>0$ for all $x \neq 0$.

(H3) there exixts a real valued function g such that $f(u)-f(v)=g(u, v)(u-v)$, for all $u \neq 0$ and $v \neq 0$ and $g(u, v) \geq L>0 \in R$.

(H4) $\phi: R \rightarrow R$ is continous for all $x \neq 0, \phi(x)>0$

(H5) $\sum_{n=M}^{\infty}(n+1) p_{n}^{2}<\infty$.

(H6) $\sum_{n=0}^{\infty} a_{n}^{2}<\infty$

(H7) $\sum_{n=0}^{\infty}(n+1) q_{n}=\infty$.

Corresponding author: I. Mohammed Ali Jaffer. 2010 Mathematics Subject Classification. 39A11.

Key words and phrases. Linear, non-linear, difference equations, oscillation and non-oscillation. 
By a solution of equation (1.1), we mean a real sequecne $\left\{x_{n}\right\}$ satsifying (1.1) for $n=$ $0,1, \ldots$ A solution $\left\{x_{n}\right\}$ of (1.1) is said to be oscillatory if it is neither eventually positve nor eventually negative. Otherwise, it is called non-oscillatory. $\Delta$ is the forward difference operator defined by $\Delta x_{n}=x_{n+1}-x_{n}$. This definition holds for other equations.

In recent years, much research is going on the study of oscillatory behavior of solutions of third order difference equations.

For more details on oscillatory behavior of difference equations, one may refer to [1-24].

\section{Main results}

In this section, we present some sufficient conditions for the oscillation of all solutions of (1.1)-(1.4). We begin with the following lemma.

Lemma 1. Let $P(n, s, x)$ be defined on $N \times N \times R^{+}, N=\{0,1,2, \ldots\}, R^{+}=[0, \infty)$, such that for fixed $n$ and s, the function $P(n, s, x)$ is non-decreasing in $x$. Let $\left\{r_{n}\right\}$ be a given sequence and the sequences $\left\{x_{n}\right\}$ and $\left\{z_{n}\right\}$ be defined on $N$ satisfying, for all $n \in N$,

$$
x_{n} \geq r_{n}+\sum_{s=0}^{n-1} P\left(n, s, x_{s}\right),
$$

and

$$
z_{n}=r_{n}+\sum_{s=0}^{n-1} P\left(n, s, z_{s}\right)
$$

respectively. Then $z_{n} \leq x_{n}$ for all $n \in N$.

The proof can be found in [15].

Theorem 1. In addition to (H1), (H2) and (H3), assume that (H5), (H6) and (H7) hold.Then,every solution of (1.1) is oscillatory.

Proof. Suppose the contrary.Then we may assume that $\left\{x_{n}\right\}$ be a non-oscillatory solution of (1.1),such that $x_{n}>0\left(\operatorname{or} x_{n}<0\right)$ for all $n \geq M-1, M>0$ is an integer.

Equation (1.1) implies

$$
\Delta\left(a_{n+1} \Delta x_{n+1}\right)-\Delta\left(a_{n} \Delta x_{n}\right)-p_{n} \Delta x_{n}+q_{n} f\left(x_{n+1}\right)=0
$$

Multiplying (2.3) by $\frac{n+1}{f\left(x_{n+1}\right)}$ and summing from $M$ to $(n-1)$, we obtain

$$
\begin{array}{r}
\sum_{s=M}^{n-1}\left(\frac{s+1}{f\left(x_{s+1}\right)}\right) \Delta\left(a_{s+1} \Delta x_{s+1}\right)-\sum_{s=M}^{n-1}\left(\frac{s+1}{f\left(x_{s+1}\right)}\right) \Delta\left(a_{s} \Delta x_{s}\right)-\sum_{s=M}^{n-1}\left(\frac{s+1}{f\left(x_{s+1}\right)}\right) p_{s} \Delta x_{s} \\
+\sum_{s=M}^{n-1}(s+1) q_{s}=0 .
\end{array}
$$


But

$$
\begin{aligned}
\sum_{s=M}^{n-1}\left(\frac{s+1}{f\left(x_{s+1}\right)}\right) \Delta\left(a_{s+1} \Delta x_{s+1}\right) & \left(\left(\frac{s+1}{f\left(x_{s+1}\right)}\right)\left(a_{s+1} \Delta x_{s+1}\right)\right)_{s=M}^{n}-\sum_{s=M}^{n-1} \Delta\left(\frac{s+1}{f\left(x_{s+1}\right)}\right)\left(a_{s+2} \Delta x_{s+2}\right) . \\
= & \frac{(n+1) a_{n+1} \Delta x_{n+1}}{f\left(x_{n+1}\right)}-\frac{(M+1) a_{M+1} \Delta x_{M+1}}{f\left(x_{M+1}\right)} \\
& -\sum_{s=M}^{n-1} \frac{a_{s+2} \Delta x_{s+2}}{f\left(x_{s+2}\right)}+\sum_{s=M}^{n-1} \frac{(s+1) a_{s+2} g\left(x_{s+2}, x_{s+1}\right) \Delta x_{s+1} \Delta x_{s+2}}{f\left(x_{s+1}\right) f\left(x_{s+2}\right)} .
\end{aligned}
$$

Also,

$$
\begin{aligned}
\sum_{s=M}^{n-1}\left(\frac{s+1}{f\left(x_{s+1}\right)}\right) \Delta\left(a_{s} \Delta x_{s}\right)= & \left(\left(\frac{s+1}{f\left(x_{s+1}\right)}\right)\left(a_{s} \Delta x_{s}\right)\right)_{s=M}^{n}-\sum_{s=M}^{n-1} \Delta\left(\frac{s+1}{f\left(x_{s+1}\right)}\right)\left(a_{s+1} \Delta x_{s+1}\right) \\
= & \frac{(n+1) a_{n} \Delta x_{n}}{f\left(x_{n+1}\right)}-\frac{(M+1) a_{M} \Delta x_{M}}{f\left(x_{M+1}\right)} \\
& -\sum_{s=M}^{n-1} \frac{a_{s+1} \Delta x_{s+1}}{f\left(x_{s+2}\right)}+\sum_{s=M}^{n-1} \frac{(s+1) a_{s+1} g\left(x_{s+2}, x_{s+1}\right)\left(\Delta x_{s+1}\right)^{2}}{f\left(x_{s+1}\right) f\left(x_{s+2}\right)} .
\end{aligned}
$$

Subsituting (2.5) and (2.6) in (2.4), we have

$$
\begin{aligned}
& \left(\frac{(n+1) a_{n+1} \Delta x_{n+1}}{f\left(x_{n+1}\right)}-\frac{(n+1) a_{n} \Delta x_{n}}{f\left(x_{n+1}\right)}\right)-\left(\sum_{s=M}^{n-1} \frac{a_{s+2} \Delta x_{s+2}}{f\left(x_{s+2}\right)}-\sum_{s=M}^{n-1} \frac{a_{s+1} \Delta x_{s+1}}{f\left(x_{s+2}\right)}\right) \\
& \quad+\left(\sum_{s=M}^{n-1} \frac{(s+1) a_{s+2} g\left(x_{s+2}, x_{s+1}\right) \Delta x_{s+1} \Delta x_{s+2}}{f\left(x_{s+1}\right) f\left(x_{s+2}\right)}-\sum_{s=M}^{n-1} \frac{(s+1) a_{s+1} g\left(x_{s+2}, x_{s+1}\right)\left(\Delta x_{s+1}\right)^{2}}{f\left(x_{s+1}\right) f\left(x_{s+2}\right)}\right) \\
& \quad-\sum_{s=M}^{n-1}\left(\frac{s+1}{f\left(x_{s+1}\right)}\right) p_{s} \Delta x_{s}+\sum_{s=M}^{n-1}(s+1) q_{s} \\
& =\left(\frac{(M+1) a_{M+1} \Delta x_{M+1}}{f\left(x_{M+1}\right)}-\frac{(M+1) a_{M} \Delta x_{M}}{f\left(x_{M+1}\right)}\right) .
\end{aligned}
$$

Using Schwarz's inequality,we have

$$
\begin{aligned}
& \sum_{s=M}^{n-1}\left(\frac{a_{s+2} \Delta x_{s+2}}{f\left(x_{s+2}\right)}\right) \leq\left(\sum_{s=M}^{n-1} a_{s+2}^{2}\right)^{\frac{1}{2}} \cdot\left(\sum_{s=M}^{n-1} \frac{\left(\Delta x_{s+2}\right)^{2}}{f^{2}\left(x_{s+2}\right)}\right)^{\frac{1}{2}} . \\
& \sum_{s=M}^{n-1}\left(\frac{a_{s+1} \Delta x_{s+1}}{f\left(x_{s+2}\right)}\right) \leq\left(\sum_{s=M}^{n-1} a_{s+1}^{2}\right)^{\frac{1}{2}} \cdot\left(\sum_{s=M}^{n-1} \frac{\left(\Delta x_{s+1}\right)^{2}}{f^{2}\left(x_{s+2}\right)}\right)^{\frac{1}{2}} . \\
& \sum_{s=M}^{n-1}\left(\frac{(s+1) a_{s+2} g\left(x_{s+2}, x_{s+1}\right) \Delta x_{s+1} \Delta x_{s+2}}{f\left(x_{s+1}\right) f\left(x_{s+2}\right)}\right) \\
& \leq\left(\sum_{s=M}^{n-1} a_{s+2}^{2}\right)^{\frac{1}{2}} \cdot\left(\sum_{s=M}^{n-1} \frac{(s+1)^{2} g^{2}\left(x_{s+2}, x_{s+1}\right)\left(\Delta x_{s+1}\right)^{2}\left(\Delta x_{s+2}\right)^{2}}{f^{2}\left(x_{s+1}\right) f^{2}\left(x_{s+2}\right)}\right)^{\frac{1}{2}} .
\end{aligned}
$$




$$
\begin{gathered}
\sum_{s=M}^{n-1}\left(\frac{(s+1) a_{s+1} g\left(x_{s+2}, x_{s+1}\right)\left(\Delta x_{s+1}\right)^{2}}{f\left(x_{s+1}\right) f\left(x_{s+2}\right)}\right) \\
\leq\left(\sum_{s=M}^{n-1} a_{s+1}^{2}\right)^{\frac{1}{2}} \cdot\left(\sum_{s=M}^{n-1} \frac{(s+1)^{2} g^{2}\left(x_{s+2}, x_{s+1}\right)\left(\Delta x_{s+1}\right)^{4}}{f^{2}\left(x_{s+1}\right) f^{2}\left(x_{s+2}\right)}\right)^{\frac{1}{2}} . \\
\sum_{s=M}^{n-1}\left(\frac{(s+1) p_{s} \Delta x_{s}}{f\left(x_{s+1}\right)}\right) \leq\left(\sum_{s=M}^{n-1}(s+1) p_{s}^{2}\right)^{\frac{1}{2}} \cdot\left(\sum_{s=M}^{n-1} \frac{(s+1)\left(\Delta x_{s}\right)^{2}}{f^{2}\left(x_{s+1}\right)}\right)^{\frac{1}{2}} .
\end{gathered}
$$

In view of (2.8), (2.9), (2.10), (2.11) and (2.12), the summation in (2.7) is bounded, we have

$$
\begin{aligned}
& \left(\frac{(n+1) a_{n+1} \Delta x_{n+1}}{f\left(x_{n+1}\right)}-\frac{(n+1) a_{n} \Delta x_{n}}{f\left(x_{n+1}\right)}\right)-\left(\sum_{s=M}^{n-1} a_{s+2}^{2}\right)^{\frac{1}{2}}\left(\sum_{s=M}^{n-1} \frac{\left(\Delta x_{s+2}\right)^{2}}{f^{2}\left(x_{s+2}\right)}\right)^{\frac{1}{2}} \\
& +\left(\sum_{s=M}^{n-1} a_{s+1}^{2}\right)^{\frac{1}{2}}\left(\sum_{s=M}^{n-1} \frac{\left(\Delta x_{s+1}\right)^{2}}{f^{2}\left(x_{s+2}\right)}\right)^{\frac{1}{2}}+\left(\sum_{s=M}^{n-1} a_{s+2}^{2}\right)^{\frac{1}{2}}\left(\sum_{s=M}^{n-1} \frac{(s+1)^{2} g^{2}\left(x_{s+2}, x_{s+1}\right)\left(\Delta x_{s+1}\right)^{2}\left(\Delta x_{s+2}\right)^{2}}{f^{2}\left(x_{s+1}\right) f^{2}\left(x_{s+2}\right)}\right. \\
& \quad-\left(\sum_{s=M}^{n-1} a_{s+1}^{2}\right)^{\frac{1}{2}}\left(\sum_{s=M}^{n-1} \frac{(s+1)^{2} g^{2}\left(x_{s+2}, x_{s+1}\right)\left(\Delta x_{s+1}\right)^{4}}{f^{2}\left(x_{s+1}\right) f^{2}\left(x_{s+2}\right)}\right)^{\frac{1}{2}}-\left(\sum_{s=M}^{n-1}(s+1) p_{s}^{2}\right)^{\frac{1}{2}}\left(\sum_{s=M}^{n-1} \frac{(s+1)\left(\Delta x_{s}\right)^{2}}{f^{2}\left(x_{s+1}\right)}\right)^{\frac{1}{2}} \\
& \leq \\
& \leq\left(\frac{(M+1) a_{M+1} \Delta x_{M+1}}{f\left(x_{M+1}\right)}-\frac{(M+1) a_{M} \Delta x_{M}}{f\left(x_{M+1}\right)}\right)-\sum_{s=M}^{n-1}(s+1) q_{s}
\end{aligned}
$$

In view of (H5), (H6) and (H8),we get from (2.13) that

$$
\begin{aligned}
\frac{(n+1)\left(a_{n+1} \Delta x_{n+1}-a_{n} \Delta x_{n}\right)}{f\left(x_{n+1}\right)} & \rightarrow-\infty \text { as } n \rightarrow \infty \\
\frac{(n+1) \Delta\left(a_{n} \Delta x_{n}\right)}{f\left(x_{n+1}\right)} & \rightarrow-\infty \text { as } n \rightarrow \infty .
\end{aligned}
$$

Hence there exists $M_{1} \geq M$ such that $\Delta\left(a_{n} \Delta x_{n}\right)<0$ for $n \geq M$

which implies $\Delta\left(a_{n} \Delta x_{n}\right)<-k, k>0$

Summing the last inequality from $m$ to $(n-1)$,we obtain

$$
\begin{aligned}
& \sum_{s=m}^{n-1} \Delta\left(a_{s} \Delta x_{s}\right)<\sum_{s=m}^{n-1}(-k) \\
& \quad\left(a_{s} \Delta x_{s}\right)_{s=m}^{n}<(-k)(n-m)
\end{aligned}
$$

(i.e) $a_{n} \Delta x_{n}<-k(n-m)+a_{m} \Delta x_{m}$

Therefore $a_{n} \Delta x_{n} \rightarrow-\infty$ as $n \rightarrow \infty$

Hence there exists

$$
M_{2} \geq M_{1} \text { such that } \Delta x_{n}<0 \text { for } n \geq M_{2}
$$

Rewriting (2.7),we have

$$
\frac{(n+1) a_{n+1} \Delta x_{n+1}}{f\left(x_{n+1}\right)}+\sum_{s=M_{2}}^{n-1} \frac{(s+1) a_{s+2} g\left(x_{s+2}, x_{s+1}\right) \Delta x_{s+1} \Delta x_{s+2}}{f\left(x_{s+1}\right) f\left(x_{s+2}\right)}
$$




$$
\begin{aligned}
= & \frac{(n+1) a_{n} \Delta x_{n}}{f\left(x_{n+1}\right)}+\frac{(M+1) a_{M+1} \Delta x_{M+1}}{f\left(x_{M+1}\right)}-\frac{(M+1) a_{M} \Delta x_{M}}{f\left(x_{M+1}\right)} \\
& -\sum_{s=M}^{n-1}(s+1) q_{s}+\sum_{s=M_{2}}^{n-1} \frac{(s+1) a_{s+1} g\left(x_{s+2}, x_{s+1}\right)\left(\Delta x_{s+1}\right)^{2}}{f\left(x_{s+1}\right) f\left(x_{s+2}\right)} \\
& -\sum_{s=M}^{M_{2}-1}\left(\frac{(s+1) a_{s+2} g\left(x_{s+2}, x_{s+1}\right) \Delta x_{s+1} \Delta x_{s+2}}{f\left(x_{s+1}\right) f\left(x_{s+2}\right)}\right)+\sum_{s=M}^{M_{2}-1}\left(\frac{(s+1) a_{s+1} g\left(x_{s+2}, x_{s+1}\right)\left(\Delta x_{s+1}\right)^{2}}{f\left(x_{s+1}\right) f\left(x_{s+2}\right)}\right) \\
& +\sum_{s=M}^{M_{2}-1}\left(\frac{a_{s+2} \Delta x_{s+2}}{f\left(x_{s+2}\right)}-\frac{a_{s+1} \Delta x_{s+1}}{f\left(x_{s+2}\right)}\right)+\sum_{s=M_{2}}^{n-1}\left(\frac{a_{s+2} \Delta x_{s+2}}{f\left(x_{s+2}\right)}-\frac{a_{s+1} \Delta x_{s+1}}{f\left(x_{s+2}\right)}\right) \\
& +\sum_{s=M_{2}}^{n-1}\left(\frac{(s+1) p_{s} \Delta x_{s}}{f\left(x_{s+1}\right)}\right)+\sum_{s=M}^{M_{2}-1}\left(\frac{(s+1) p_{s} \Delta x_{s}}{f\left(x_{s+1}\right)}\right)
\end{aligned}
$$

From (H1), (H8), (??) and (2.14), there exists an integer $M_{3} \geq M_{2}$, such that

$$
\frac{(n+1) a_{n+1} \Delta x_{n+1}}{f\left(x_{n+1}\right)}+\sum_{s=M_{2}}^{n-1} \frac{(s+1) a_{s+2} g\left(x_{s+2}, x_{s+1}\right) \Delta x_{s+1} \Delta x_{s+2}}{f\left(x_{s+1}\right) f\left(x_{s+2}\right)} \leq-k, k \geq M_{3}
$$

where $k$ is a positive constant.

$$
\frac{-(n+1) a_{n+1} \Delta x_{n+1}}{f\left(x_{n+1}\right)}-\sum_{s=M_{2}}^{n-1} \frac{(s+1) a_{s+2} g\left(x_{s+2}, x_{s+1}\right) \Delta x_{s+1} \Delta x_{s+2}}{f\left(x_{s+1}\right) f\left(x_{s+2}\right)} \geq k
$$

let $u_{n+1}=-(n+1) \Delta x_{n+1}$, (2.15) becomes

$$
\begin{aligned}
& \frac{u_{n+1} a_{n+1}}{f\left(x_{n+1}\right)} \geq k+\sum_{s=M_{3}}^{n-1} \frac{(s+1) a_{s+2} g\left(x_{s+2}, x_{s+1}\right) \Delta x_{s+1} \Delta x_{s+2}}{f\left(x_{s+1}\right) f\left(x_{s+2}\right)} ; n \geq M_{3} \\
& \Rightarrow u_{n+1} \geq k \frac{f\left(x_{n+1}\right)}{a_{n+1}}+\sum_{s=M_{3}}^{n-1} \frac{a_{s+2} f\left(x_{n+1}\right) g\left(x_{s+2}, x_{s+1}\right)\left(-\Delta x_{s+2}\right) u_{s+1}}{a_{n+1} f\left(x_{s+1}\right) f\left(x_{s+2}\right)}
\end{aligned}
$$

Also,

$$
\text { Let } v_{n+1}=k \frac{f\left(x_{n+1}\right)}{a_{n+1}}+\sum_{s=M_{3}}^{n-1} \frac{a_{s+2} f\left(x_{n+1}\right) g\left(x_{s+2}, x_{s+1}\right)\left(-\Delta x_{s+2}\right) v_{s+1}}{a_{n+1} f\left(x_{s+1}\right) f\left(x_{s+2}\right)}
$$

Using Lemma 1, we have, from (2.16) and (2.17)

$$
\Rightarrow u_{n+1} \geq v_{n+1}
$$

(2.16) implies

$$
v_{n+1}=\frac{f\left(x_{n+1}\right)}{a_{n+1}}\left(k+\sum_{s=M_{3}}^{n-1} \frac{a_{s+2} g\left(x_{s+2}, x_{s+1}\right)\left(-\Delta x_{s+2}\right) v_{s+1}}{f\left(x_{s+1}\right) f\left(x_{s+2}\right)}\right)
$$

This implies

$$
v_{n+1} \geq \frac{k f\left(x_{M_{3}}\right)}{a_{n+1}} \text { for } n \geq M_{3}
$$


From (2.19) and (2.20),we have

$$
\begin{aligned}
-(n+1) \Delta x_{n+1} \geq \frac{k f\left(x_{M_{3}}\right)}{a_{n+1}} & \\
& \Rightarrow \Delta x_{n+1} \leq \frac{-k f\left(x_{M_{3}}\right)}{(n+1) a_{n+1}}
\end{aligned}
$$

Summing (2.20) from $M_{3}$ to $(n-1)$

$$
\begin{aligned}
\sum_{s=M_{3}}^{n-1} \Delta x_{s+1} & \leq-k f\left(x_{M_{3}}\right) \sum_{s=M_{3}}^{n-1} \frac{1}{(s+1) a_{s+1}} \\
\left(x_{s+1}\right)_{s=M_{3}}^{n} & \leq-k f\left(x_{M_{3}}\right) \sum_{s=M_{3}}^{n-1} \frac{1}{(s+1) a_{s+1}} \\
& \Rightarrow x_{n+1} \leq x_{M_{3}+1}-k f\left(x_{M_{3}}\right) \sum_{s=M_{3}}^{n-1} \frac{1}{(s+1) a_{s+1}} \\
& \Rightarrow x_{n} \leq 0 \text { for sufficiently large } n,
\end{aligned}
$$

which yields a contradiction to the fact that $x_{n}$ is eventually positive.The proof is similar for the case when $x_{n}$ is eventually negative. Hence the theorem is completely proved.

Example 1. Consider the difference equation

$$
\Delta^{2}\left(\frac{1}{n} \Delta x_{n}\right)-\frac{9 n^{2}+18 n+5}{2 n(n+1)(n+2)} \Delta x_{n}+\frac{n^{2}+2 n+1}{n(n+1)(n+2)} x_{n+1}=0
$$

All the conditions of theorem 1 are satisfied .Hence every solution of equation(E1) is oscillatory.

One such solution of (2.21) is $x_{n}=(-1)^{n}$.

Example 2. Consider the difference equation

$$
\Delta^{2}\left(\frac{1}{n+1} \Delta x_{n}\right)-\frac{n^{4}+7 n^{3}+35 n^{2}+92 n+45}{(n+1)(n+2)(n+3)} 2^{n+1} \Delta x_{n}+(n+1) 8^{n+1} x_{n+1}^{3}=0
$$

All the conditions of Theorem 1 are satisfied .Hence every solution of equation (2.22) is oscillatory.

One such solution of (2.22) is $x_{n}=\frac{(-1)^{n}}{2^{n}}$.

Corollary 1. In addition to (H1), (H2), (H3) and (H4), assume that (H5), (H6) and (H7) hold. Then, every solution of (1.2) is oscillatory.

Example 3. Consider the difference equation

$$
\Delta^{2}\left(\frac{1}{2 n}\left(x_{n}^{4}\right) \Delta x_{n}\right)-\frac{3 n^{4}+9 n^{3}+14 n^{2}+16 n+4}{4 n(n+1)(n+2)} \Delta x_{n}+n\left(x_{n+1}^{3}+\frac{x_{n+1}^{5}}{2}\right)=0
$$

All the conditions of Corollary 1 are satisfied .Hence every solution of equation (2.23) is oscillatory.

One such solution of (2.23) is $x_{n}=(-1)^{n+1}$. 
Remark 1. In Corollary 1, assume $\phi\left(x_{n}\right)=c>0$, a constant, in (1.2) yields (1.1).

Corollary 2. In addition to $(\mathrm{H} 1),(\mathrm{H} 2)$ and $(\mathrm{H} 3)$, assume that $(\mathrm{H} 6)$ and $(\mathrm{H} 7)$ hold. Then, every solution of (1.3) is oscillatory.

Example 4. Consider the difference equation

$$
\Delta^{2}\left(\frac{1}{n} \Delta x_{n}\right)+\frac{2(3 n+2)}{n(n+1)(n+2)} x_{n+1}^{5}=0
$$

All the conditions of Corollary 2 are satisfied. Hence every solution of equation (2.24) is oscillatory.

One such solution of (2.24) is $x_{n}=(-1)^{n+1}$.

Corollary 3. In addition to (H1), (H2), (H4) and (H4), assume that (H6) and (H7) hold. Then, every solution of (1.4) is oscillatory.

Example 5. Consider the difference equation

$$
\Delta^{2}\left(\frac{1}{2 n+1}\left(x_{n}^{2}\right) \Delta x_{n}\right)+\frac{8(n+1)}{(n+1)^{3}\left(n^{2}+2 n+3\right)}\left(x_{n+1}^{3}+\frac{x_{n+1}^{5}}{2}\right)=0
$$

All the conditions of Corollary 3 are satisfied .Hence every solution of equation (2.25) is oscillatory.

One such solution of (2.25) is $x_{n}=n(-1)^{n}$.

Proofs of Corollary 1, Corollary 2 and Corollary 3 are simillar to the proof of Theorem 1 and hence the details are omitted.

\section{References}

[1] R. P. Agarwal, Difference Equation and Inequalities-Theory, Methods and Applications, 2nd edition.

[2] R. P. Agarwal, Martin Bohner, Said R. Grace and Donal O'Regan, Discrete Oscillation Theory, CMIA Book Series, Volume 1, ISBN: 977-5945-19-4.

[3] R. P. Agarwal, Mustafa F. Aktas and A. Tiryaki, On oscillation criteria for third order nonlinear delay differential equations, Archivum Mathematicum(BANO)-Tomus 45 (2009), 1-18.

[4] W. T. Li and R. P. Agarwal, Interval oscilation critical for second order non linear differential equations with damping, Comp. Math. Appl., 40 (2000), 217-230.

[5] John R. Greaf and E. Thandapani, Oscillatory and asymptotic behavior of solutions of third order delay diiference equations, Funkcialaj Ekvacioj, 42(1999), 355-369.

[6] Said. R. Grace, Ravi O. Agarwal and John R. Greaf, Oscllation criteria for certain third order nonlinear difference equations, Appl. Anal. Discrete. Math., 3(2009), 27-28.

[7] S. H. Saker, Oscillation of third order difference equations, Portugaliae Mathematica-Vol 61 Fasc3-2004-Nova series.

[8] Sh. Salem and K. R. Raslam, Oscillation of some second order damped difference equations, IJNS 5 (2008), 246-254. 
[9] B. Selvaraj and I. Mohammed ali jaffer, Oscillation theorems of solutions for certain third order functional difference equations with dealy-bulletin of pure and applied sciences, (to appear).

[10] B. Selvaraj and I. Mohammed ali jaffer, Oscillatory properties of fourth order neutral delay difference equations, Journal of Computer and Mathematical Sciences-An International Research Journal, 1 (2010), 364373.

[11] B. Selvaraj and I. Mohammed ali jaffer, Oscillation behavior of certain third order non-linear difference equations, International Journal of Nonlinear Science (to appear).

[12] B. Selvaraj and J. Daphy Louis Lovenia, Oscillation Behavoir of Fourth Order Neutral Difference Equations with variable coefficients, Far East Journal of Mathematical Sciences, 35 (2009), 225-231.

[13] B. Selvaraj and I. Mohammed ali jaffer, Oscillation behavior of certain third order linear difference equations, Far East Journal of Mathematical Sciences, 40, (2010), 169-178.

[14] E. Thandapani and B. S. Lalli, Oscillations Criteria for a Second Order Damped Difference Equations, Appl. Math. Lett. 8 (1995), 1-6.

[15] E. Thandapani, I. Gyori and B. S. Lalli, An application of discrete inequality to second order non-linear oscillation, J. Math. Anal. Appl., 186(1994), 200-208.

[16] E. Thandapani and S. Pandian, On the oscillatory behavior of solutions of second order non-linear difference equations, ZZA 13(1994), 347-358.

[17] E. Thandapani and S. Pandian, Oscillation theorem for non- linear second order difference equations with a non linear damping term, Tamkang J. Math.

[18] E. Thandapani, Asymptopic and oscillatory behavior of solutions of non linear second order difference equations, Indian J. Pure and Appl. Math., 24(1993), 365-372.

[19] E. Thandepani and K. Ravi, Oscilation of second order half linear difference equations, Appl. Math. Lett., 13 (2000), 43-49.

[20] E. Thandapani and B. Selvaraj, Existence and Asymtopic behavior of non oscillatory Solutions of certain Nonlinear Difference Equation, Far East Journal of Mathematical Sciences, 14(2004), 9-25.

[21] E. Thandapani and B. Selvaraj, Oscillatory behavior of solutions of three dimentional delay difference system, Radovi Mathematicki, 13(2004), 39-52.

[22] E. Thandapani and B. Selvaraj, Oscillatory and non-oscillatory behavior of fourth order quasi-linear difference equation, Far East Journal of Mathematical Sciences, 17(2004), 287-307.

[23] E. Thandapani and B. Selvaraj, Behavior of oscillatory and non oscillatory solutions of certain fourth order quasi-linear difference equations, The Mathematical Equations, $\mathbf{X X X I X ( 2 0 0 5 ) , ~ 2 1 4 - 2 3 2 . ~}$

[24] E. Thandapani and B. Selvaraj, Oscillation of fourth order quasi-linear difference equation, Fasci culi Mathematici Nr 37(2007), 109-119.

Research Scholar, Department of Mathematics, Karunya University, Coimbatore, Tamil Nadu, India.

E-mail: jaffermathsgac@gmail.com

Dean of Science and Humanities, Nehru Institute of Engineering and Technology, Coimbatore, Tamil Nadu, India. 